\title{
Adult Lymphoblastic Lymphoma
}

National Cancer Institute

\section{Source}

National Cancer Institute. Adult Lymphoblastic Lymphoma. NCI Thesaurus. Code C9361.

A lymphoblastic lymphoma occurring in adults. 